\title{
Stability of meridionally-flowing grounded abyssal currents in the ocean
}

\author{
G. E. Swaters \\ Applied Mathematics Institute, \\ Department of Mathematical \& Statistical Sciences and \\ Institute for Geophysical Research, University of Alberta, Edmonton, \\ Canada
}

\begin{abstract}
Deep western boundary currents are an important component of the thermohaline circulation in the ocean, which plays a dominant role in Earth's evolving climate. By exploiting the underlying Hamiltonian structure, sufficient stability (and hence necessary instability) conditions are derived for meridionally-flowing grounded abyssal currents, based on a baroclinic model corresponding to a low frequency limit of the three-layer shallow water equations on a $\beta$-plane with variable topography.
\end{abstract}

Keywords: abyssal overflows, deep western boundary currents, meridional overturning circulation, baroclinic instability, climate dynamics.

\section{Introduction}

In a source region of deep water formation, the Sverdrup vorticity balance predicts equatorward abyssal flow (Stommel and Arons [1]). Away from the source region, Stommel-Arons theory cannot infer the flow direction of abyssal currents. However, many abyssal currents are characterized by the isopycnal field being grounded against sloping topography and the flow being in geostrophic balance. As shown by Nof [2], a fully grounded abyssal water mass lying over sloping topography flows, in the fully nonlinear but reduced gravity dynamical limit, nondispersively and steadily in the along slope direction, irrespective of the specific height or vorticity field within the abyssal water mass.

These two results provide a dynamical scenario for the initiation and maintenance of source-driven grounded abyssal flow. That is, in high latitude regions 
where deep water is produced (often over sloping topography), the Sverdrup vorticity balance initiates equatorward flow. Once produced, this water mass can become grounded and geostrophically adjusted, maintaining a Nof balance that permits sustained basin scale meridional quasi-steady and coherent abyssal flow. The principal purpose of the present contribution is to briefly present the stability properties associated with a baroclinic model that describes the dynamics of grounded abyssal currents as process that begins as a source-driven "StommelArons flow" and then transitions to a "Nof flow" as the abyssal water mass flows equatorward. The report given here is part of a much larger study on the dynamics on grounded abyssal flow by Swaters $[3,4]$.

\section{Governing equations}

The model is a low frequency (sub-inertial) limit of the three-layer shallow-water equations on a $\beta$-plane with variable topography (for full details see Swaters [3]). In this limit, the primary dynamical variables are the geostrophic (leading order reduced) pressures in the upper two layers and the thickness of the bottom or abyssal layer (see Fig. 1). The nondimensional governing equations are given by

$$
\begin{gathered}
{\left[\partial_{t}+J\left(\phi_{1}, \cdot\right)\right]\left[\Delta \phi_{1}-F_{1}\left(\phi_{1}-\phi_{2}\right)+\beta y\right]=\frac{F_{1} Q}{F_{1}+F_{2}}+\frac{\triangle^{2} \phi_{1}}{R_{e}},} \\
{\left[\partial_{t}+J\left(\phi_{2}, \cdot\right)\right]\left[\Delta \phi_{2}-F_{2}\left(\phi_{2}-\phi_{1}\right)+h+h_{B}+\beta y\right]} \\
=-r_{2} \Delta \phi_{2}+\frac{F_{1} Q}{F_{1}+F_{2}}+\frac{\triangle^{2} \phi_{2}}{R_{e}}, \\
h_{t}+J\left(h+\phi_{2}+h_{B}, \frac{h}{1+s \beta y}\right)=Q+r_{3} \triangle\left(\phi_{2}+h_{B}+h\right),
\end{gathered}
$$

with the auxiliary diagnostic relations

$$
\left.\begin{array}{c}
\mathbf{u}_{1,2}=\mathbf{e}_{3} \times \nabla \phi_{1,2}, \mathbf{u}_{3}=\frac{\mathbf{e}_{3} \times \nabla\left(\phi_{2}+h_{B}+h\right)}{1+s \beta y}, \\
p=\phi_{2}+h_{B}+h, \eta=\phi_{2}-\phi_{1},
\end{array}\right\}
$$

with $J(A, B)=A_{x} B_{y}-A_{y} B_{x}$, and where the 1,2 or 3 subscript on a physical variable refers to the upper, middle and abyssal layer, respectively, alphabetical subscripts (unless otherwise noted) indicate partial differentiation, $\mathbf{u}_{1,2,3}=\left(u_{1,2,3}, v_{1,2,3}\right), \nabla=\left(\partial_{x}, \partial_{y}\right), \Delta=\nabla \cdot \nabla, h_{B}$ is the height of the topography, $h$ is the height of the abyssal layer relative to $h_{B}, \eta$ is the deflection (measured positively upward) of the interface between the two upper layers from its equilibrium position and $Q$ is the down or upwelling term, respectively. The dynamic pressures in the upper two layers is given by $\phi_{1,2}$, and in the abyssal layer by $p$, respectively. Equations $(4 \mathrm{c}, \mathrm{d})$ express the continuity of total pressure across the deforming interfaces between the middle and abyssal layers and the upper 


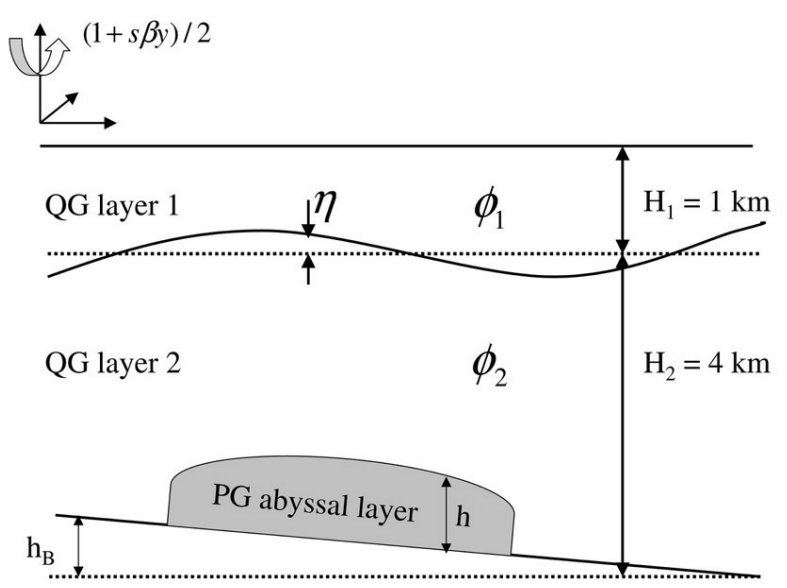

Figure 1: Model geometry used in this paper.

and middle layers, respectively. The model is a hybrid quasigeostrophic/planetary geostrophic (QG/PG) system in which (1) and (2) are the QG and (3) is the PG potential vorticity equations, respectively, for the individual layers.

The dynamical parameters are defined by

$$
s=\frac{s^{*} L}{H_{2}}, \beta=\frac{\beta^{*} L^{2}}{U_{*}}, R_{e}=\frac{U_{*} L}{A_{H}}, F_{1}=\frac{g^{\prime} H_{2}}{\widetilde{g} H_{1}}, F_{2}=\frac{g^{\prime}}{\widetilde{g}}, r_{2,3}=\frac{r_{2,3}^{*}}{s H_{2}},
$$

where $H_{1,2}$ are the constant reference layer thicknesses in the upper two layers, $\rho_{*}$ is the reference Boussinesq density and $g^{\prime}=\left(\rho_{3}-\rho_{2}\right) g / \rho_{*}>0$ and $\tilde{g}=\left(\rho_{2}-\right.$ $\left.\rho_{1}\right) g / \rho_{*}>0$ where $\rho_{1,2,3}$ correspond to the constant density in each individual layer with $0<\rho_{1}<\rho_{2}<\rho_{3}, L=\sqrt{g^{\prime} H_{2}} / f_{0}$ (the internal deformation radius for the middle layer), $U_{*}=s^{*} g^{\prime} / f_{0}$ (the Nof speed), $s^{*} \approx O\left(\nabla^{*} h_{B}^{*}\right.$ ) (a representative value for the topographic slope), $f_{0}$ is the reference Coriolis parameter, $\beta^{*}$ is the northward gradient of the Coriolis parameter, respectively. In addition, $A_{H}$ is the horizontal eddy coefficient in the upper two layers and $r_{2,3}^{*}$ are "bottom friction coefficients" for the middle and abyssal layers, respectively. Ekman boundary layer theory implies that $r_{2,3}^{*}=H_{2,3} \sqrt{E_{2,3}^{V}}$, where $H_{2,3}$ are the vertical thickness scales and $E_{2,3}^{V}$ are the vertical Ekman numbers for, respectively, the middle and abyssal layers. Accordingly, $r_{2,3}^{*}$ are the scale vertical thicknesses of the Ekman bottom boundary layer in the middle and abyssal layers, respectively.

The unforced, inviscid dynamics of the model is purely baroclinic (i.e., the horizontal divergence of the barotropic mass flux is zero). Although there is no "thermodynamics" in the model per se (so that there is no genuine heat or salinity transport), $Q>0$ can be heuristically interpreted as a cooling of the overlying water column that leads to a downward mass flux resulting in the depletion of the 
overlying water mass and corresponding increase in the volume of abyssal water. Similarly, $Q<0$ can be heuristically interpreted as warming or freshening of the abyssal water mass that leads to an upward mass flux resulting in the depletion of the abyssal water mass and a corresponding increase in the volume of upper ocean water.

\section{Stability problem}

To examine the baroclinic instability problem in its simplest form, but still retain the most important features, the inviscid, rigid-lid and $2 \frac{1}{2}$-layer approximation $F_{1,2}=\phi_{1}=r_{2,3}=0, R_{e} \rightarrow \infty$, is introduced into (1), (2) and (3), yielding

$$
\begin{gathered}
(\Delta \eta+h)_{t}+J\left(\eta, \Delta \eta+h+h_{B}+\beta y\right)=0, \\
h_{t}+J\left(h+\eta+h_{B}, \frac{h}{1+s \beta y}\right)=Q,
\end{gathered}
$$

where, for convenience, $\eta \equiv \phi_{2}$.

The area integrated energy for (6) and (7) is given by

$$
\mathcal{E}(\eta, h)=\frac{1}{2} \iint_{\Omega} \nabla \eta \cdot \nabla \eta+\left(h+h_{B}\right)^{2}-h_{B}^{2} d x d y,
$$

which will be invariant in the inertial limit $Q=0$. If $Q=0,(6)$ and (7) is a $2 \times 2$ infinite dimensional Hamiltonian dynamical system (Swaters $[5,6]$ ), in which $\mathcal{E}$ will be the Hamiltonian functional and the Hamiltonian variables are given by $\Delta \eta+h$ and $h$, respectively. The Casimirs (i.e., the set of invariant functionals that span the Kernel of the Poisson bracket; see Swaters [5]), which are needed in the variational principle, may be written in the form

$$
\begin{gathered}
\mathcal{C}_{1}=\iint_{\Omega}\left\{\int_{h_{B}+\beta y}^{\Delta \eta+h+h_{B}+\beta y} \digamma_{1}(\xi) d \xi\right\} d x d y, \\
\mathcal{C}_{2}=\iint_{\Omega}(1+s \beta y)\left\{\int_{0}^{h /(1+s \beta y)} \digamma_{2}(\xi) d \xi\right\} d x d y,
\end{gathered}
$$

where $\digamma_{1,2}$ are arbitrary functions of their arguments.

\subsection{Steady solutions, variational principle and stability conditions}

General inertial steady abyssal solutions to (6) and (7) of the form

$$
\eta=\tilde{\eta}=0, h=\tilde{h}(x, y), h_{B}=h_{B}(x, y), Q=0,
$$

must satisfy

$$
J\left(\tilde{h}+h_{B}, \frac{\tilde{h}}{1+s \beta y}\right)=0 \Longrightarrow \widetilde{h}+h_{B}=\digamma\left(\frac{\tilde{h}}{1+s \beta y}\right),
$$

for some function $\digamma$. 
The solution (12) satisfies the first order conditions for an extremal of the invariant functional

$$
\mathcal{I} \equiv \mathcal{E}+\mathcal{C}_{1}+\mathcal{C}_{2}=\mathcal{E}-\iint_{\Omega}(1+s \beta y)\left\{\int_{0}^{h /(1+s \beta y)} \digamma(\xi) d \xi\right\} d x d y,
$$

where $\digamma_{1}=0$ and $\digamma_{2}=-\digamma$. It follows (assuming $\eta=0$ on the boundary of $\Omega$ ) that

$$
\begin{aligned}
\delta \mathcal{I}(\eta, h) & =\iint_{\Omega}-\eta \Delta \delta \eta+\left[h+h_{B}-\digamma\left(\frac{h}{1+s \beta y}\right)\right] \delta h d x d y \\
& =\iint_{\Omega}-\eta(\Delta \delta \eta+\delta h)+\left[h+\eta+h_{B}-\digamma\left(\frac{h}{1+s \beta y}\right)\right] \delta h d x d y,
\end{aligned}
$$

so that $\delta \mathcal{I}(\tilde{\eta}, \tilde{h})=0$.

The second variation of $\mathcal{I}$ evaluated at the steady solution $(\tilde{\eta}, \tilde{h})$ is given by

$$
\begin{aligned}
\delta^{2} \mathcal{I}(\tilde{\eta}, \tilde{h}) & =\iint_{\Omega} \nabla \delta \eta \cdot \nabla \delta \eta+\left[1-\frac{\digamma^{\prime}\left(\frac{\tilde{h}}{1+s \beta y}\right)}{(1+s \beta y)}\right](\delta h)^{2} d x d y, \\
& =\iint_{\Omega} \nabla \delta \eta \cdot \nabla \delta \eta-\frac{h_{B_{x}}(x, y)}{\widetilde{h}_{x}(x, y)}(\delta h)^{2} d x d y,
\end{aligned}
$$

where the "prime" means differentiation with respect to the argument and (12) has been used. It is always the case that $\delta^{2} \mathcal{I}(\tilde{\eta}, \widetilde{h})$ is an invariant of the linear stability equations (Swaters [6]). Since the integrand of the functional $\delta^{2} \mathcal{I}(\widetilde{\eta}, \tilde{h})$ is a diagonalized quadratic form with respect to $(\delta \eta, \delta h)$, if $\delta^{2} \mathcal{I}(\widetilde{\eta}, \widetilde{h})$ is definite in sign for all perturbations, then $(\tilde{\eta}, \tilde{h})$ is linearly stable (in the sense of Liapunov with respect to the norm $\left.\left[\left|\delta^{2} \mathcal{I}(\tilde{\eta}, \tilde{h})\right|\right]^{\frac{1}{2}}\right)$.

The case where $\delta^{2} \mathcal{I}(\widetilde{\eta}, \widetilde{h})<0$ is not considered. It requires certain mathematical properties to hold on the domain $\Omega$ and while these can occur this analysis is not pursued (see Swaters [5] for the $f$-plane Hamiltonian-based analysis). The case where $\delta^{2} \mathcal{I}(\tilde{\eta}, \widetilde{h})>0$ is precisely analogous to Fjørtoft's stability theorem (Swaters [6]) and reduces to the $f$-plane results of Swaters [5, 7].

It follows from $(15)$ that $\delta^{2} \mathcal{I}(\widetilde{\eta}, h)>0$ when

$$
\frac{h_{B_{x}}(x, y)}{\widetilde{h}_{x}(x, y)} \leq 0,
$$

This is a sufficient condition for stability. A necessary condition for instability is, therefore, that there exists at least one point $(x, y) \in \Omega$ for which

$$
\frac{h_{B_{x}}(x, y)}{\widetilde{h}_{x}(x, y)}>0 .
$$

Even though $\beta$ has been fully retained, this stability condition is identical in form to that obtained by Swaters [7]. 
Consider the case where $h_{B_{x}}(x, y)<0$ as would occur, on average, along the western shelf-slope region of an ocean basin (see Fig. 1). The necessary condition for instability is that there exists at least one point for which $\widetilde{h}_{x}(x, y)<0$. For a parabolically shaped abyssal current with up slope and down slope groundings, this condition holds on the down slope flank but not on the up slope flank. This is why the instability preferentially amplifies on the down slope flank and the amplitude of the perturbations along the down slope grounding are much larger compared to those on the up slope grounding (see Swaters $[4,7,8]$ ). Physically, energy is required to move grounded abyssal fluid parcels located adjacent to the up slope grounding up the sloping bottom (against the force of gravity), while energy is released by the down slope movement of grounded abyssal fluid parcels located along the down slope grounding. The result is that there is a spatial asymmetry (even on an $f$-plane) in the destabilization of these grounded abyssal currents. This asymmetry is clearly seen in numerical simulations (Swaters [4, 8]).

\section{Baroclinic instability characteristics for a constant velocity abyssal current}

The general linear stability equations are analytically intractable. However, much can be learned from the constant velocity abyssal flow on the linearly sloping bottom, given by

$$
h_{0}=\tilde{h}-\gamma x \geq 0, h_{B}=-x,
$$

where $\tilde{h}>0$ is constant, and neglecting terms of $O(s \beta)$ but retaining $\beta$. The abyssal height (18) is the simplest profile for $h_{0}$ that satisfies the necessary condition for instability and for which the stability problem can be solved explicitly.

The linear stability problem associated with (18) can be written in the form

$$
\Delta \eta_{t}+\beta \eta_{x}+(h+\eta)_{y}=0, h_{t}-h_{y}+\gamma \eta_{y}=0
$$

These equations will be solved in the meridional channel domain $x \in(0, L)$ so that the appropriate boundary condition is $\eta=0$ on $x=0, L$. It is convenient to write the solution in the form

$$
(h, \eta)=A[\gamma /(1+c), 1] \sin (n \pi x / L) \exp [i k(y-c t)-i \beta x /(2 c k)]+c . c .,
$$

where $n \in \mathbb{Z}^{+}$, c.c. means the complex conjugate of the preceding term, $k$ is the meridional, or along slope, wavenumber, $A$ is a free amplitude constant, and $c$ is the complex-valued phase velocity that must satisfy the dispersion relationship

$$
K^{2} c^{3}+\left(1+K^{2}\right) c^{2}+\left[1+\gamma-(\beta / 2 k)^{2}\right] c-(\beta / 2 k)^{2}=0,
$$

where $K \equiv \sqrt{k^{2}+l^{2}}$ is the wavenumber modulus and $l \equiv n \pi / L$.

The three roots to the cubic dispersion relation (21) correspond to a barotropic and baroclinic topographic Rossby wave and to a planetary Rossby wave, respectively (Swaters [3]). The onset of instability corresponds to the coalescence of 
the barotropic and baroclinic topographic Rossby modes. Mathematically, this coalescence occurs when the discriminate for the cubic (21) is zero and this condition defines the marginal stability boundary. Thus, the marginal stability boundary is given by

$$
\varsigma_{1}^{3}-\left(\varsigma_{2} \varsigma_{1} / 2\right)^{2}-9 \varsigma_{1} \varsigma_{2} \varsigma_{3} / 2+27 \varsigma_{3}^{2} / 4+\varsigma_{2}^{3} \varsigma_{3}=0
$$

where

$$
\varsigma_{1} \equiv\left[1+\gamma-(\beta / 2 k)^{2}\right] / K^{2}, \varsigma_{2} \equiv 1+1 / K^{2}, \varsigma_{3} \equiv-[\beta /(2 k K)]^{2} .
$$

Equation (22) is itself a cubic with respect to $\varsigma_{1}$ (i.e., $\gamma$ ). It can be shown that the discriminate for (22) is strictly positive since $\varsigma_{2}>0$ and $\varsigma_{3}<0$ and is zero only if $\beta=0$. Thus, there is only one real solution for $\gamma$, denoted as $\gamma_{c}(k, l, \beta)$, and this is the marginal stability boundary (or, equivalently, the critical equatorward abyssal velocity). That is, for a given $k, l$ and $\beta$, instability only occurs if $\gamma>\gamma_{c}$, (neutral) stability occurs if $\gamma \leq \gamma_{c}$ and the marginal stability boundary is given by $\gamma=\gamma_{c}(k, l, \beta)$. The point of marginal stability will be the minimum $\gamma_{c} \geq 0$, with respect to $k$ and $l$, for fixed $\beta$. In the limit $\beta=0$, (22) reduces to $\gamma_{c}=\left(K^{2}-1\right)^{2} /\left(4 K^{2}\right)$, which is the $f$-plane result of Mooney and Swaters [9]. Based on the nondimensionalizations introduced, it is possible to characterize the most unstable mode as having an along slope wavelength on the order of $94 \mathrm{~km}$, an equatorward phase velocity on the order of $3 \mathrm{~cm} / \mathrm{s}$, a modal period on the order of 38 days and an $e$-folding amplification time on the order of 6 days (Swaters [3]).

\section{Conclusions}

A model has been presented capable of describing the meridional flow of source driven grounded abyssal flow in a stratified ocean. In the inviscid, unforced limit, the model can be written as an infinite-dimensional noncanonical Hamiltonian dynamical system and this formalism has been exploited to establish sufficient linear stability (and hence necessary instability) conditions for general steady bottom-intensified abyssal flow. The linear stability equations were explicitly solved in the case of an equatorward flowing abyssal current with constant velocity. The most unstable mode, which corresponds to a baroclinic topographic planetary wave, has a wavelength on the order of $94 \mathrm{~km}$, an equatorward phase velocity on the order of $3 \mathrm{~cm} / \mathrm{s}$, a modal period on the order of 38 days and an $e$-folding amplification time on the order of 6 days.

\section{Acknowledgement}

Preparation of this extended abstract was supported in part by the Natural Sciences and Engineering Research Council of Canada. 


\section{References}

[1] Stommel, H., and Arons, A. B., On the abyssal circulation of the world ocean I. Stationary flow patterns on a sphere. Deep-Sea Res., 1960, 6, 140-154.

[2] Nof, D., The translation of isolated cold eddies on a sloping bottom. Deep-Sea Res., 1983, 30, 171-182.

[3] Swaters, G. E., The meridional flow of source-driven abyssal currents in a stratified basin with topography. Part I. Model development and dynamical properties. J. Phys. Oceanogr., 2006, 36, 335-355.

[4] Swaters, G. E., The meridional flow of source-driven abyssal currents in a stratified basin with topography. Part II. Numerical Simulation. J. Phys. Oceanogr., 2006, 36, 356-375.

[5] Swaters, G. E., Nonlinear stability of intermediate baroclinic flow on a sloping bottom. Proc. R. Soc. Lond. A, 1993, 442, 249-272.

[6] Swaters, G. E., Introduction to Hamiltonian Fluid Dynamics and Stability Theory, 2000 (Chapman\&Hall/CRC Press: New York).

[7] Swaters, G. E., On the baroclinic instability of cold-core coupled density fronts on sloping continental shelf. J. Fluid Mech., 1991, 224, 361-382.

[8] Swaters, G. E., Numerical simulations of the baroclinic dynamics of densitydriven coupled fronts and eddies on a sloping bottom. J. Geophys. Res., 1998, 103, 2945-2961.

[9] Mooney, C. J., and Swaters, G. E., Finite amplitude baroclinic instability of a mesoscale gravity current in a channel. Geophys. Astrophys. Fluid Dyn., 1996, 82, 173-205. 